\title{
Swept source optical coherence tomography Gabor fusion splicing technique for microscopy of thick samples using a deformable mirror
}

\author{
Christopher Costa, ${ }^{\text {a }}$ Adrian Bradu, ${ }^{\text {a }}$ John Rogers, ${ }^{\text {a }}$ Pauline Phelan, ${ }^{\text {b }}$ Adrian Podoleanu, ${ }^{\text {a }}$ \\ ${ }^{a}$ University of Kent, Applied Optics Group, School of Physical Sciences, Canterbury, United Kingdom, CT2 7NZ \\ $\mathrm{b}_{\text {University of Kent, Cell Biology, Cancer Targets and Therapies Group, School of BioSciences, Canterbury, United }}$ \\ Kingdom, CT2 7NZ
}

\begin{abstract}
In this paper we present a swept source optical coherence tomography (OCT) system at $1060 \mathrm{~nm}$ equipped with a wavefront sensor at $830 \mathrm{~nm}$ and a deformable mirror in a closed loop adaptive optics (AO) system. Due to the $\mathrm{AO}$ correction, the confocal profile of the interface optics becomes narrower than the OCT axial range, restricting the part of the B-scan (cross-section) with good contrast. By actuating on the deformable mirror, the depth of the focus is changed and the system is used to demonstrate Gabor filtering in order to produce B-scan OCT images with enhanced sensitivity throughout the axial range from a Drosophila larvae. The focus adjustment is achieved by manipulating the curvature of the deformable mirror between two user-defined limits. Particularities of controlling the focus for Gabor filtering using the deformable mirror are presented.
\end{abstract}

Keywords: optical coherence tomography, confocal microscopy, adaptive optics.

Address all correspondence to: Christopher Costa, University of Kent, Applied Optics Group, School of Physical Sciences, Canterbury, United Kingdom CT2 7NZ; Tel: +44 1227 7643772; E-mail: cc375@kent.ac.uk

\section{Introduction}

Adaptive optics $(\mathrm{AO})^{1}$ have been used to great effect in astronomy, eye imaging ${ }^{2}$ and confocal microscopy (CM) systems by exploiting the capability to reduce system- and sample-induced optical aberrations to enhance resolution. ${ }^{3}$ Adding AO to an optical coherence tomography (OCT) set-up enables further reduction of the voxel size of the imaging system, producing a much smaller 3D volume per voxel due to enhanced transversal resolution, in addition to axial. ${ }^{4,5}$ Additionally, swept source OCT (SS-OCT) can provide exceptional imaging speeds due to the rate at which the wavelength can be tuned. ${ }^{6,7}$ The combination of these technologies have produced fast imaging systems capable of very high resolution, ideally suited for traditionally difficult clinical applications such as ophthalmology. 


\subsection{Problems presented with AO/SS-OCT}

There is a trend in developing long coherence length swept sources that can determine an axial range extending over $1 \mathrm{~cm}$ and more. ${ }^{9}$ On the other hand, an $\mathrm{AO}$ enhanced confocal microscope system can shrink the confocal profile width to values of tens of micrometers or even smaller. As the SS-OCT method presents the disadvantage that the reflectance of all points along the axial range are measured under a fixed focus, maximum sensitivity within the cross-sectional (B-scan) OCT image is achieved in the focus only. If the full width half maximum (FWHM) of the confocal profile, acting as a confocal gate (CG) is smaller than the OCT axial range (AR), then the OCT image presents high contrast and good transversal resolution within a spatially restricted stripe determined by the CG only.

\subsection{Possible solution}

The problem presented is that the region of highest quality (as determined by contrast and resolution) is limited by the width and position of the CG. Efforts have been made to extend the depth of focus (DOF) whilst maintaining high lateral resolution. One approach uses an axicon lens to produce a Bessel beam, extending the DOF when compared with a Gaussian beam. The DOF is enlarged at the expense of lowered sensitivity, making the method less suitable for imaging samples of low reflectance ${ }^{10,11}$ although progress has been made to improve its efficiency. ${ }^{12}$

A solution was developed based on Gabor filtering ${ }^{13}$ that shifts the CG incrementally through the sample; an OCT cross-sectional image was acquired at 5 different focus positions (R) as manipulated by an electrical lens. From each of the 5 images, the in-focus region was extracted and then spliced together to form a single image wherein all regions are in focus.

It was determined that 5 repetitions of data acquisition with shifted focus were sufficient as the 
CG profile was approximately $1 / 5$ th of the AR. Due to the enhanced coherence length of swept sources, the ratio $\mathrm{R}=\mathrm{AR} / \mathrm{CG}$ may be much larger than 5, in which case the Gabor procedure may slow the acquisition considerably. This presents a problem as the process is largely manual, with a margin for error increasing as there may be variations in the imaging parameters over time, especially with living samples. The next logical step would be the design of a system that simplifies and expedites this procedure.

\section{Methodology}

As detailed in Fig. 1, an AO configuration is presented where a deformable mirror (DM), normally employed by the AO closed loop to correct the wavefront, is also used to shift the focus for Gabor filtering. A DM was already reported as the element for focus control in a time domain OCT (TD-OCT) system. ${ }^{14}$ The focus was moved in synchronism with the coherence gate, achieving what is known as dynamic focus. ${ }^{15}$ This procedure is not applicable in spectral domain OCT. In addition, in a closed loop AO configuration, attempts of focus control have to be disconnected from the feedback loop operation, which actively works to reduce aberrations (including defocus). In a related report, ${ }^{16}$ an $\mathrm{AO}$ system using a DM combined with spectrometer based OCT was used to control the focus position and shape of the confocal gate by actuating on the spherical aberration introduced by the DM itself. Here we actuate on a different aberration component, defocus, and use such control to implement Gabor filtering as detailed below.

\subsection{Concept}

Given the speed of SS-OCT imaging, which allows frame rates as high as $100 \mathrm{~Hz}$, there is room for sufficient high values of $\mathrm{R}$ to still secure video rate, which is important in live imaging systems. ${ }^{17}$ 


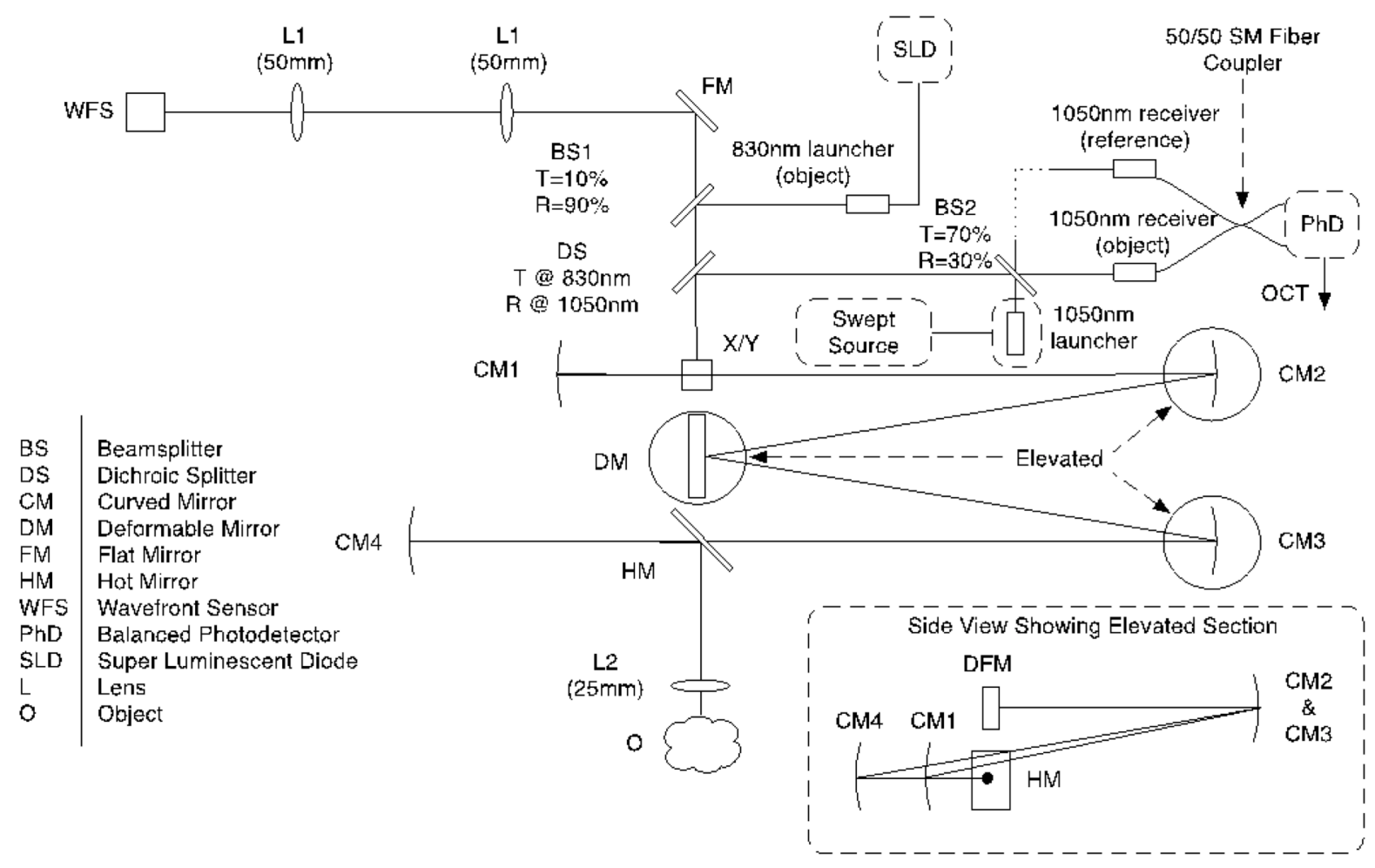

Fig 1 Schematic of the AO/SS-OCT system with imaging at $1060 \mathrm{~nm}$ and wavefront sensing plus confocal microscopy at $830 \mathrm{~nm}$.

R B-scans are acquired where the region of maximum intensity as determined by the CG profile is shifted deeper within the object along the $\mathrm{z}$ coordinate. After acquisition, each image obtained in this way may then be cropped to isolate the in-focus regions in depth. The cropped images are subsequently assembled together across $\mathrm{R}$ into a single synthesized image of uniform intensity.

The focus control using a DM is achieved by generating a set of corrections by controlling the magnitude of the Zernike polynomial responsible for that of defocus only. Closed loop correction will be conducted to optimize the wavefront, providing a base configuration for the DM. The focus will then be shifted and the closed loop executed again to achieve optimization at the new position. This will provide two states (base state and modified state) from which intermediate positions may be interpolated. In essence, we would have a set of states where the corrections have been optimized for imaging at different axial positions in the sample. 


\subsection{Optical design for minimizing system-induced aberrations}

This implementation of DM control utilizes a system of passive and active aberration correction to improve image quality. Passive correction is achieved through an optimized system wherein the number of optical elements (specifically the curved mirrors required to resize the beam, known for inducing aberrations) has been minimized. Collimators (Schafter \& Kirchhoff: FC-F-4-M15-37) launch beams of $3 \mathrm{~mm}$ diameter.

By using the two galvoscanners, $\mathrm{X}$ and $\mathrm{Y}(5 \mathrm{~mm})$, close to each other, the number of curved mirrors is reduced. Two telescopes (pairs of curved mirrors with different focal lengths) are placed in the setup to increase the beam diameter to $15 \mathrm{~mm}$ to fully cover the reflective surface of a DM (Imagine Optics: Mirao-52e), then to reduce the beam down to $7.5 \mathrm{~mm}$ on the final lens (L2) of 25 $\mathrm{mm}$ focal length.

Furthermore, these telescopes are arranged in a non-planar configuration with the beam propagating in a single plane, either horizontal or vertical. This is seen in Fig. 1 where the beam travels between curved mirrors CM1 and CM2 in the plane Y, with no deviation in X. The beam is then propagated horizontally across the surface of the DM and then vertically to CM4, at the same elevation with CM1. This technique has been shown to compensate for astigmatism aberrations induced by reflections from curved mirrors. ${ }^{18}$ We measured sensitivity of the OCT setup to be 100 $\mathrm{dB}$ using previously reported techniques. ${ }^{19}$ The NA of the system was calculated to be 0.175 . For imaging we use a $100 \mathrm{kHz}$ Axsun swept source with a central wavelength at $1060 \mathrm{~nm}$.

We determine the field of view experimentally by blocking the reference arm (using the confocal signal only) to image a large bar on the USAF resolution target. For this measurement, the DC output of one photodetector from the balanced detector was used. The target is mounted on 
an XYZ translation stage, allowing movement of the bar to the limits of the displayed image and measurement of the displacement using the micrometer screws. From this we determine that for an amplitude of $1 \mathrm{~V}$ of driving signals applied to both scanners we achieve a $700 \times 700 \mu \mathrm{m}^{2}$ raster size, and with $0.1 \mathrm{~V}$ the area is $70 \times 70 \mu \mathrm{m}^{2}$.

\subsection{Active aberration compensation using deformable mirror}

Active wavefront correction is achieved using a single 52-actuator MEMS DM in conjunction with a Shack-Hartmann sensor (Imagine Optics: HASO-32). Wavefront sensing is performed using light from a $830 \mathrm{~nm}$ broadband super luminescent diode (SLD), whose beam path is almost completely shared with the $1060 \mathrm{~nm}$ imaging arm. Prior to imaging, using a scattering target such as paper, correction is achieved that brings the DM into an optimal starting configuration where any residual aberrations induced by the optical elements in the system itself are reduced.

With the inclusion of tilts and focus in the wavefront error calculations, we are able to achieve a wavefront RMS value better than $0.02 \mu \mathrm{m}$. After a stable correction is achieved, the voltage values applied to the DM are saved into an XML file. This file contains a $1 \mathrm{D}$ array of 52 numerical values, each corresponding to a single actuator on the DM. The values of this array represent the voltage settings of the 52 actuators on the DM that achieves the best wavefront correction. This set is considered a base state, i.e. the midpoint of the axial imaging range to be used next.

\subsection{Shifting the confocal gate}

The next stage is to modify the amplitude of a single Zernike polynomial, in this case that of defocus is selected, to a different value. This causes the mirror to adjust its shape to add defocus aberration in addition to its corrected state. With defocus added, the closed loop is executed again 
to compensate for the possible increase in other aberrations caused by manipulation of the focus. No other types of aberrations are directly added in this way, so it can be assumed that the difference between the original base state and the modified state is the addition of defocus only. Again, the modified state file is a 1D array of 52 values, this time representing the position of every actuator on the DM to form a state with modified focus. This modified state file is also saved alongside the original base state file.

Given that the linearity of the DM is greater than $95 \%,{ }^{20}$ the difference between the base state and the modified state is a linear increase in defocus aberration. Custom software programmed in LabVIEW dynamically interpolates a 2D array of intermediate voltage values for every actuator using these two $1 \mathrm{D}$ arrays. The size of the $2 \mathrm{D}$ array is $\mathrm{R}$ by 52 , where $\mathrm{R}$ is the number of steps required. Each successive column in this $2 \mathrm{D}$ array contains a complete set of 52 actuator voltage values, representing an incremental shift in focus.

This 2D array can be expanded to any size and any range of focus, limited only by the speed and stroke of the DM, respectively. A larger array may impact the rate at which the DM actuates the commands. Increasing the size of the array (number of columns) will adjust the number of incremental steps (R) between two user-defined limits. Shifting the confocal gate by $40 \mu \mathrm{m}$ in 50 steps covers a $2 \mathrm{~mm}$ range as determined experimentally using a mirror as an object and finding the new focus position for the deformed state of the DM.

Adjusting the limits of the array affects the magnitude of defocus aberration applied to the DM, and in this way the AR can be adjusted dynamically while scanning to suit the sample thickness. To produce a smooth focus sweep at a consistent rate, we typically make these limits symmetrical, i.e. between -1 and $1 \mathrm{~mm}$ with the midpoint representing zero defocus added. The visible effect on the image is a sweeping region of high intensity through the depth range of the sample. 


\subsection{Analysis of the confocal profile and signal strength during focus sweep}

It is important to determine how the confocal profile is affected as we shift the focus through the sample. To test the imaging performance we set the DM to assume the base state corrected configuration. During the measurement we set the AO to run in open loop, i.e. the corrections are maintained without feedback from the WFS. In this mode we may still directly manipulate the mirror to add defocus.

A mirror is mounted on a XYZ translation stage and placed as the object in the imaging arm. We block the reference arm and use the confocal signal (with weak light emitted from the SLD to avoid saturating the detector) to determine the confocal profile of the system at this default state. The DM is then set to add different values of defocus in increments of $1 \mu \mathrm{m}$ RMS. For each new focus adjustment, the translation stage with the mirror was moved around the focussed position and the strength of the confocal signal was measured to construct the confocal gate profile.

Confocal profiles for three focus adjustments are shown in Fig. 2. From the graphs we can ascertain two critical facts about the focus sweep. Firstly, it is clear that the image intensity drops as the DM introduces more defocus.

Secondly, it is important to note that the width of the profile increases with defocus. We start with a FWHM of $60 \mu \mathrm{m}$ in the base state, which first spreads to a FWHM $70 \mu \mathrm{m}$ as defocus aberration is increased to $1 \mu \mathrm{m}$ RMS, then spreads further to FWHM $80 \mu \mathrm{m}$ with $2 \mu \mathrm{m}$ RMS defocus. The enlargement of the confocal profile and reduction of its maximum with the defocus applied suggests deterioration of the correction. Naturally this would decrease the depth resolution of the confocal microscope (the object arm), though this is not a concern with OCT imaging.

The sensitivity decay with defocus is quantified for larger defocus values in Fig. 3. In this case, 


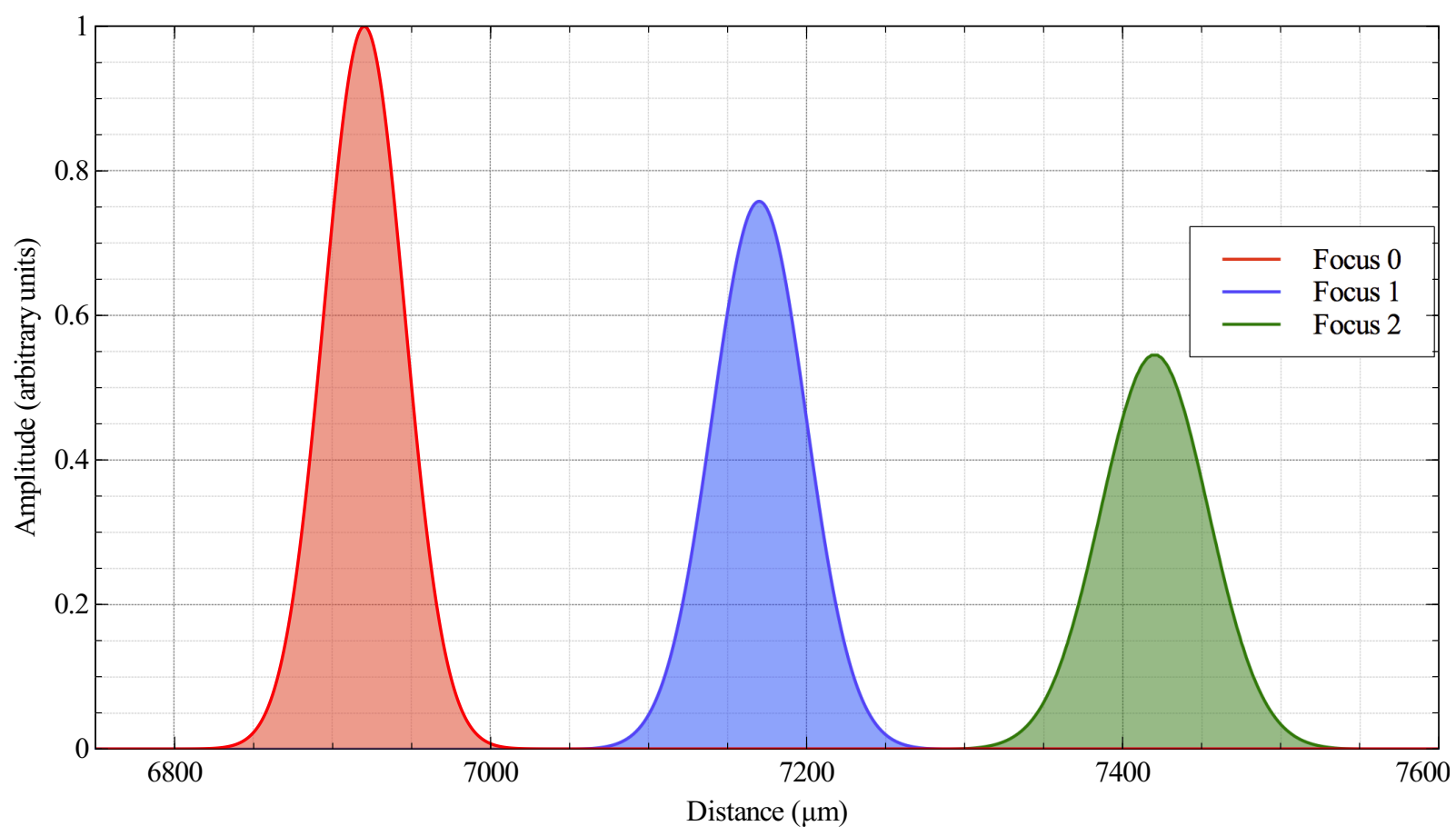

Fig 2 Chart showing how the confocal profile shifts in depth and changes shape as we add 1 and $2 \mu$ m RMS focus.

for each defocus value applied by the DM, the object mirror was moved back into focus using the translation stage and the strength of the confocal signal was measured. There appears to be a sharp drop as the focus first moves from base state, then recorded intensity decreases below $50 \%$ for $\pm 3 \mu \mathrm{m}$ RMS defocus, reaching $10 \%$ for $\pm 8 \mu \mathrm{m}$ RMS defocus. The power profile is shown as symmetric around base state when adding positive or negative focus.

The chart in Fig. 3 can be used to select a useful range to operate the DM where the sensitivity loss can be tolerated for a given imaging object. This sensitivity loss is a limitation of this technique, not observed with other methods of focus control such as using an electrical lens.

From the data shown in Fig. 2, it is clear that applying $1 \mu \mathrm{m}$ RMS defocus equates to an axial shift of the focus by $250 \mu \mathrm{m}$, therefore a sweep between $-2 \mu \mathrm{m}$ RMS and $2 \mu \mathrm{m}$ RMS would offer a $1 \mathrm{~mm}$ axial imaging range. 


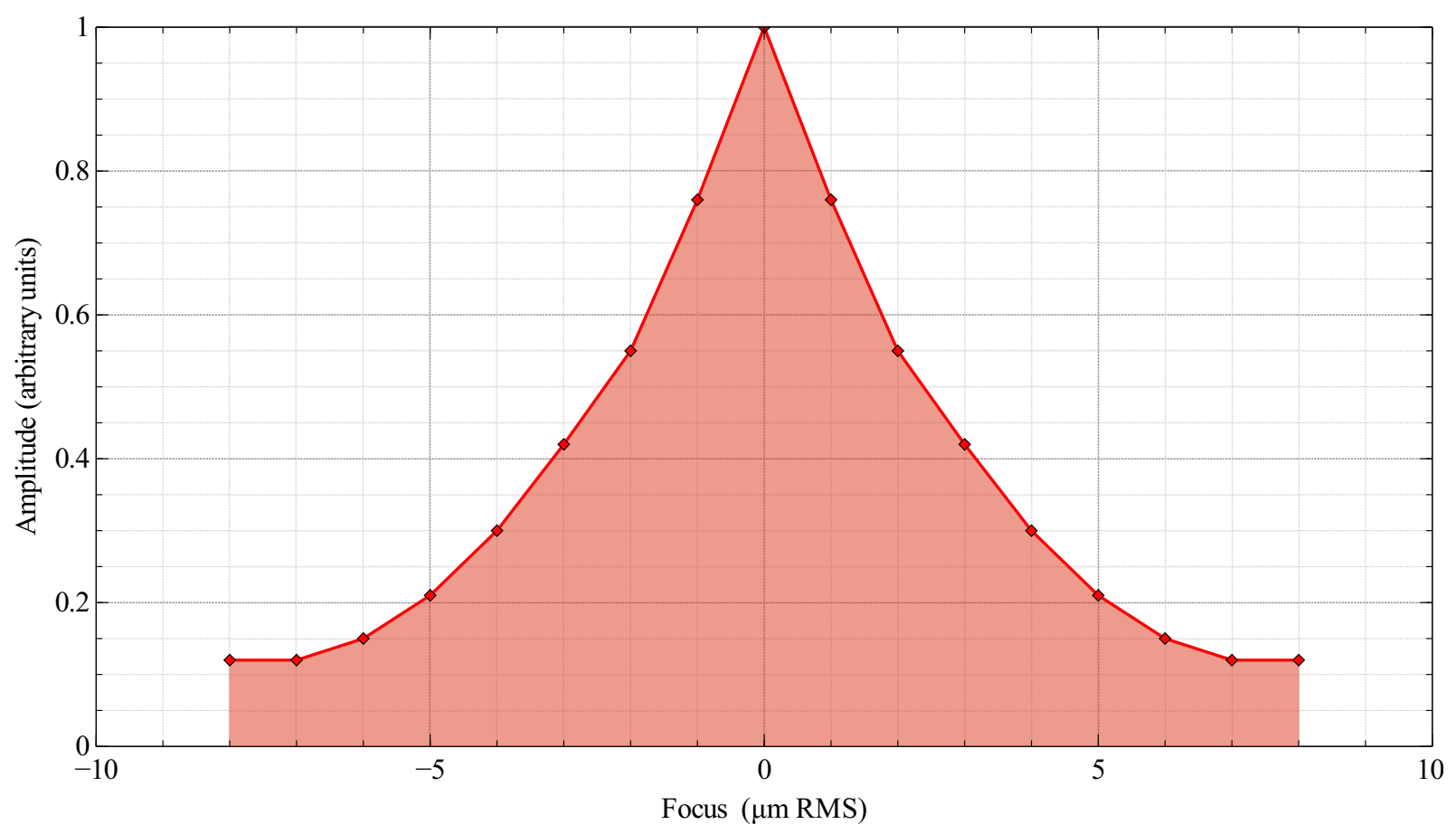

Fig 3 Chart showing decay in signal intensity with added focus

\subsection{Assessment of the transversal resolution}

To test practical image resolution, an image is acquired of a USAF 1951 target, as shown in Fig.

4. We know from the data shown in section 2.2 that for driving signals of $0.1 \mathrm{~V}$ amplitude applied to both scanners we achieve a scan area of $70 \times 70 \mu \mathrm{m}^{2}$ on the sample. Given an image size of $250 \times 250$ pixels, it can be deduced that each pixel covers $0.28 \mu \mathrm{m}$ along horizontal and vertical directions.

Fig. 4 shows an image of the USAF target where two sets of three bars (each $\sim 2 \mu \mathrm{m}$ apart) are shown distributed both vertically and horizontally. Given the parameters described above, we may analyze the distance between the bars in pixels to determine the actual spacing in $\mu \mathrm{m}$. Both the horizontal and vertical bars are shown to be 7 pixels apart: $7 * 0.32=1.96 \mu \mathrm{m}$ apart. This confirms our reported transversal resolution values.

To determine how lateral resolution is affected when axially shifting the CG, the USAF 1951 


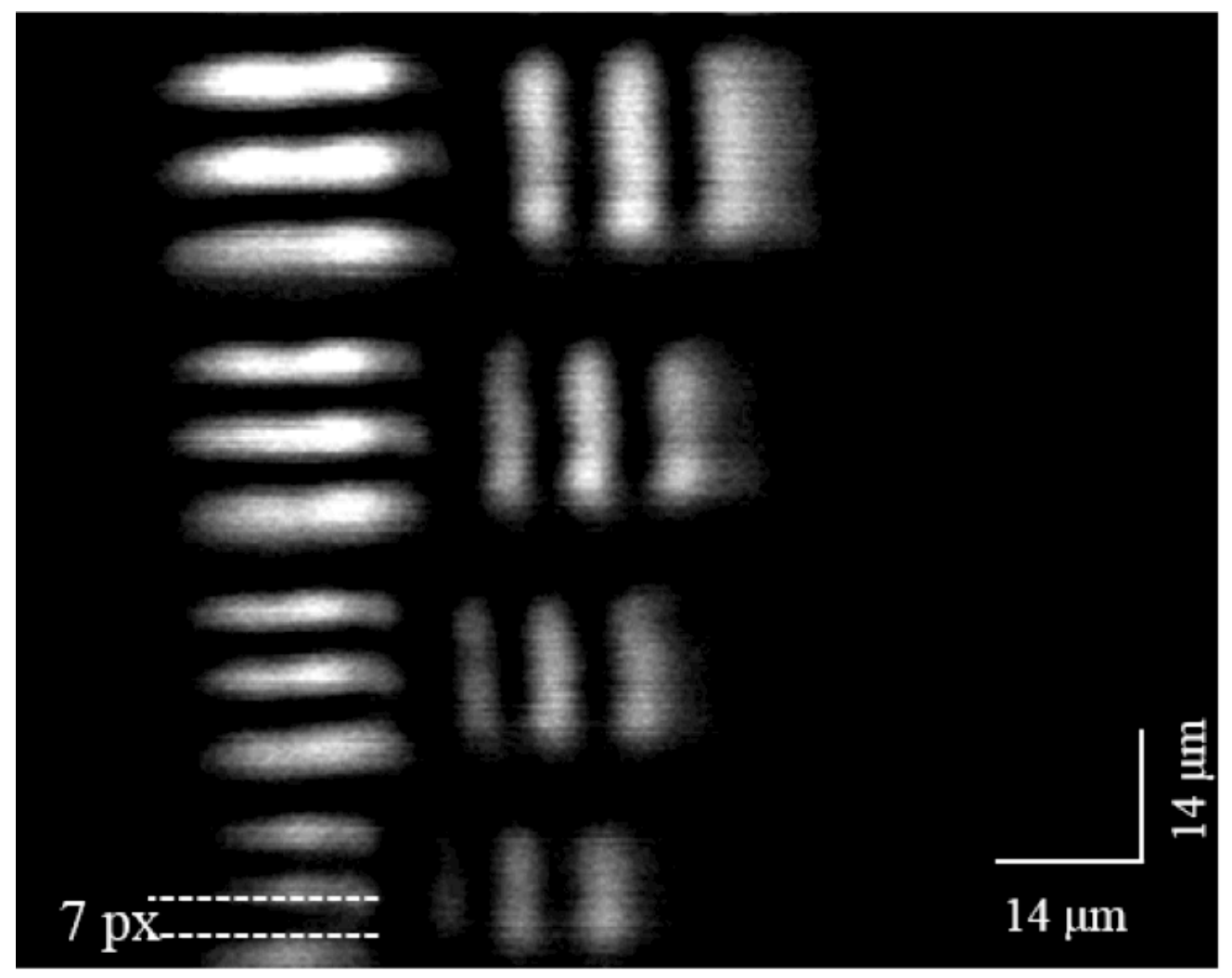

Fig 4 High contrast confocal image of the USAF target showing the smallest $2 \mu \mathrm{m}$ bars at the bottom. Two sets of three bars are shown oriented horizontally and vertically. Image size is 250 x 250 pixels covering an actual area of 70 $\mathrm{x} 70 \mu \mathrm{m}^{2}$ on the target.

resolution target is used as the object and images are acquired for different defocus values. Initially we assume the base state with no defocus, and position the object such that the set containing the smallest bars (of $2 \mu \mathrm{m}$ spacing) are within the centre of the frame. Fig. 5 shows four additional images taken of the same region of the target, with different levels of defocus applied to the DM. Each time defocus is applied to the DM, the USAF target is manually moved axially back into focus before acquiring the image. The distance between the bars in the images obtained with focus -1 , focus 1 and focus 2 (focus moved at $-250 \mu \mathrm{m},+250 \mu \mathrm{m}$ and $+500 \mu \mathrm{m}$ respectively) are the same as in the image at focus 0 . There appears to be a loss in signal intensity in the image acquired with focus $-2(-500 \mu \mathrm{m})$, which is not apparent in the others, but the transversal resolution seems intact. 


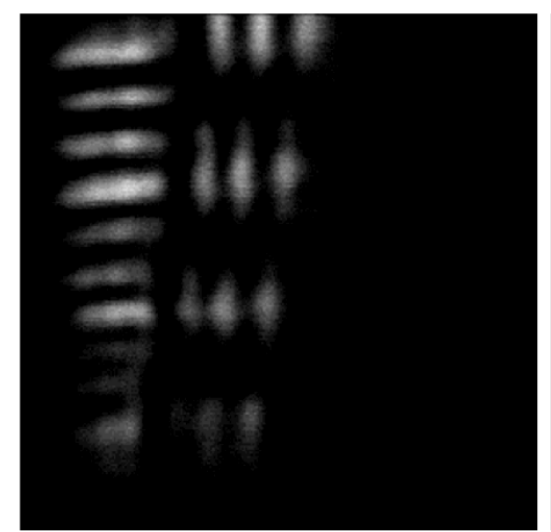

(A)

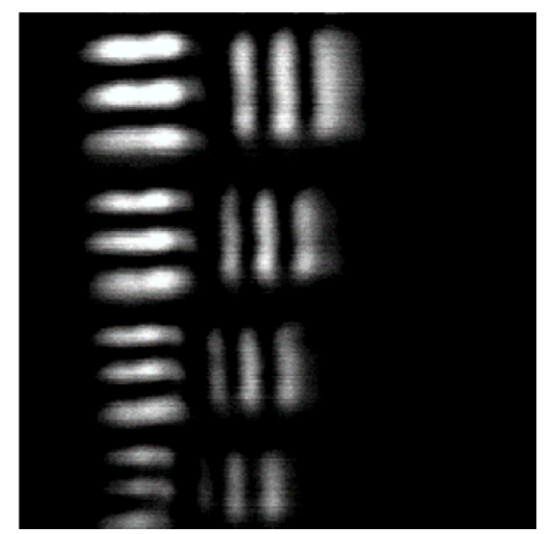

(D)

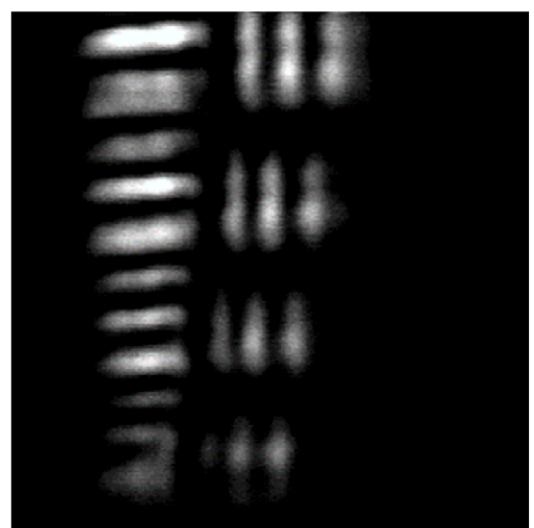

(B)

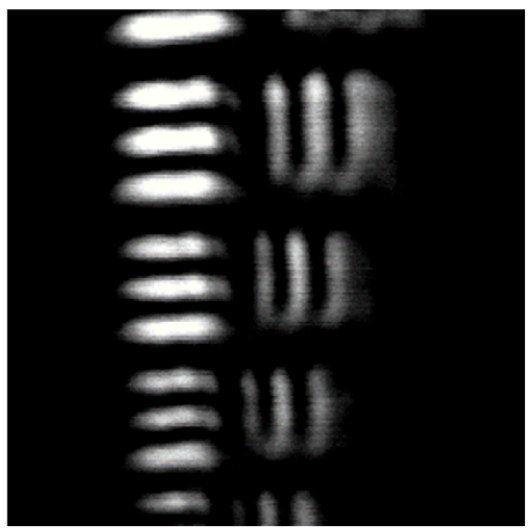

(E)

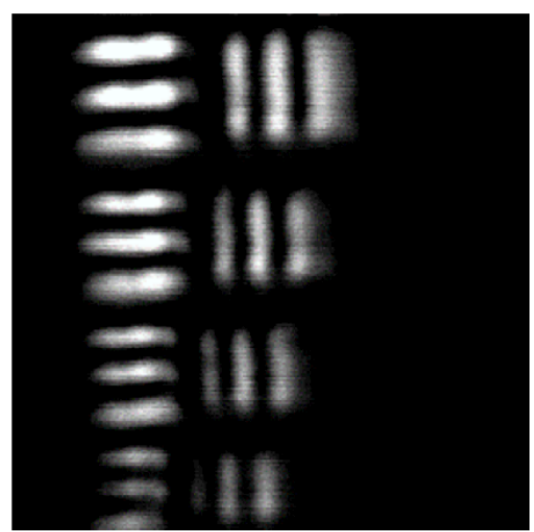

(C)

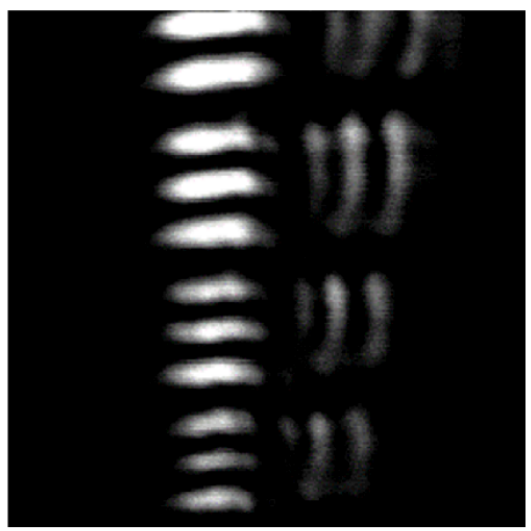

(F)

Fig 5 Effect of defocus on the image acquired from the USAF target, using the same area as in Fig. 4. The original image (C) is repeated in (D) and placed alongside images taken with -2 (A), -1 (B), 1 (E) and 2 (F) $\mu \mathrm{m}$ RMS focus added to the DM.

Some lateral drift is also identified, seen by the bars moving across the image as the focus is changed. From $5 \mathrm{C}$ to $5 \mathrm{~B}$, the bars moved up by 7 pixels and from $5 \mathrm{~B}$ to $5 \mathrm{~A}$ by further 7 pixels. The same, from 5D to 5E, the bars moved down by 7 pixels, and from $5 \mathrm{E}$ to $5 \mathrm{~F}$, by further 7 pixels. 7 pixels vertical shift means $2 \mu \mathrm{m}$, i.e. comparable and larger than the transversal resolution. In terms of horizontal shift of the image, from $5 \mathrm{C}$ to $5 \mathrm{~B}$ there is a slight shift of 1 pixel, while from $5 \mathrm{~B}$ to $5 \mathrm{~A}$ of 7 pixels to the left. From 5D to 5E, there is a lateral shift to the right of 7 pixels and from $5 \mathrm{E}$ to $5 \mathrm{~F}$ an additional 7 pixels to the right.

The experiments reported in section 2.5 show that the variation of focus imprinted by the DM when obtaining the images in Fig. 5 covers a $1 \mathrm{~mm}$ axial range. Using the same process described 
above, the effect of applying larger defocus values to the DM is evaluated by the resolution in the images acquired, shown in Fig. 6.

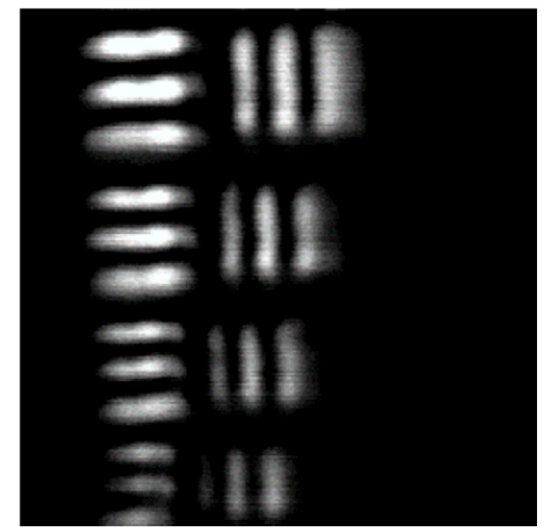

(A)

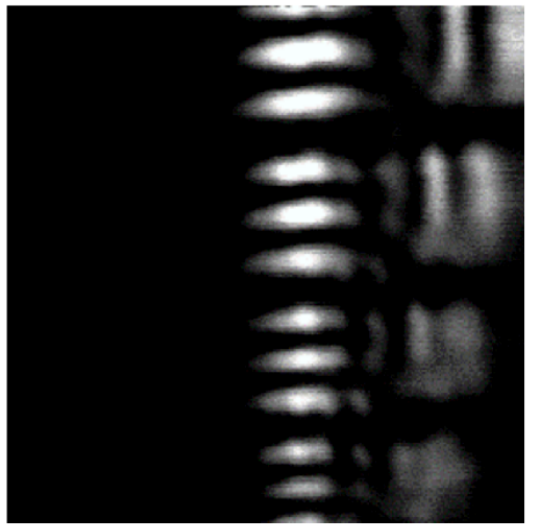

(B)

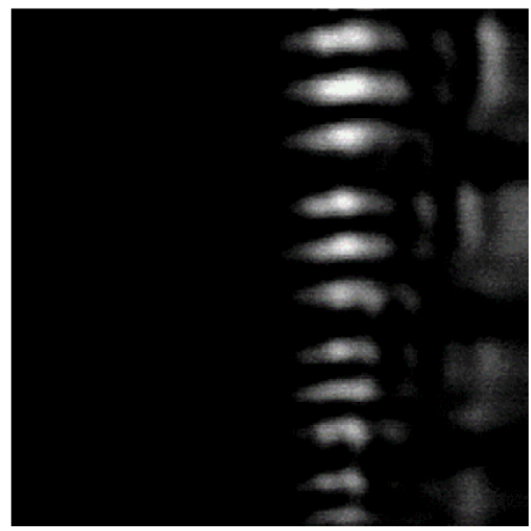

(C)

Fig 6 Images of the USAF target obtained using the DM deformed to create $3 \mu \mathrm{m}$ (B) and $4 \mu \mathrm{m}$ (C) RMS defocus, with the original $0 \mu \mathrm{m}$ focus image (A) for comparison.

In the image in Fig. 6B, the bars moved to the right by 24 pixels (top of the bar barely visible), i.e. by $6.9 \mu \mathrm{m}$. In the image in $6 \mathrm{C}$, the bars moved to the right by 28 pixels, i.e. by $8 \mu \mathrm{m}$. In terms of vertical shift, the bars moved down in $6 \mathrm{~B}$ by 21 pixels and in $6 \mathrm{C}$ by 28 pixels. On average, from images in Fig. 5 and 6, it can be inferred that each $1 \mu \mathrm{m}$ defocus shifts the image down and to the right by approximately 7 pixels and each $-1 \mu \mathrm{m}$ defocus shifts the image up and to the left by approximately 7 pixels, i.e. $2 \mu \mathrm{m}$ or a resolution interval.

In conclusion, RMS focus control via the DM in the configuration assembled has a pronounced effect on the lateral drift. It is important to note here that the lateral resolution as determined by the horizontal bars appears to remain unaffected, though the vertical bars have become distorted and blurry. Practically, good signal is obtained from the central part of the image only. We believe this is due to the fact that the correction file was taken with the scanners in a zeroed position, meaning the corrections are less valid for the periphery of the imaged area.

Some lateral shift is expected due to the off axis configuration, typical in AO systems using 
DMs. This can be further reduced by increasing the focal length of the spherical mirrors CM2 and $\mathrm{CM} 3$, with the obvious disadvantage of enlarging the size of the layout and reduction in the axial range covered. Another factor is the imaging beam potentially being slightly off-axis to the USAF target. A combination of these two factors may be responsible for the slight horizontal shift between image in Fig. 5B and 5C. We noticed that by tilting the USAF target, the lateral shifts change. This prevents post processing compensation by software.

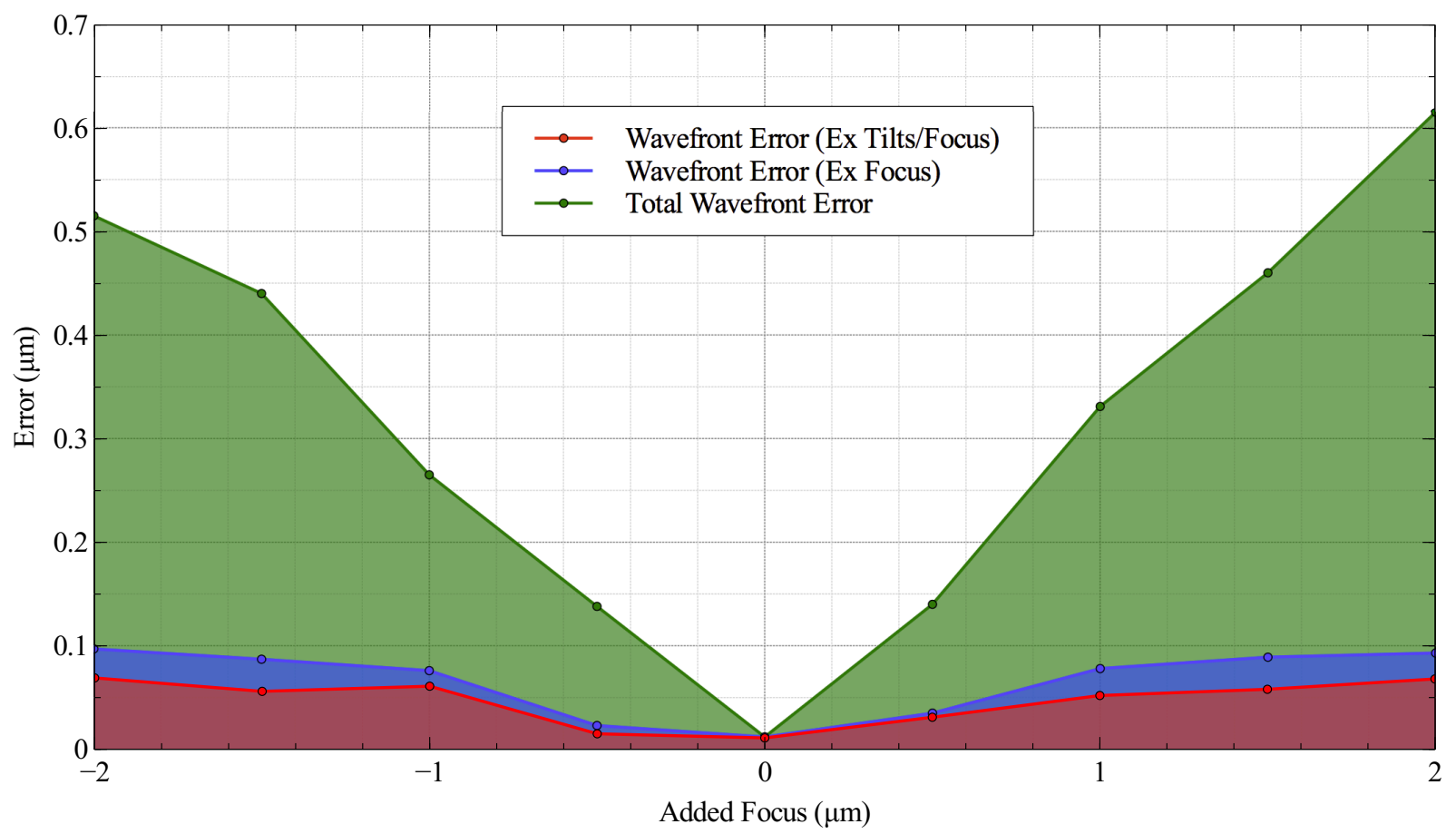

Fig 7 RMS wavefront error as focus is manipulated. Total error: green region. Error excluding focus: blue region. Error excluding tilts and focus: red region.

\subsection{Effect upon wavefront when manipulating focus}

In-depth analysis of the effect of focus manipulation on other aberrations was conducted. WFS measurements are taken as the DM is set to varying levels of defocus. Fig. 7 shows the overall wavefront error as detected by the WFS, with focus varied between -0.5 and $0.5 \mathrm{~mm}$. It can be observed that tilt contributes significantly to the overall wavefront error in comparison to the other 
aberrations. It can also be inferred from the chart that the total wavefront error excluding defocus is 5 times less than the value with defocus. This demonstrates that the contribution of defocus to wavefront error is much greater than the aberrations that increase as a consequence of focus manipulation.

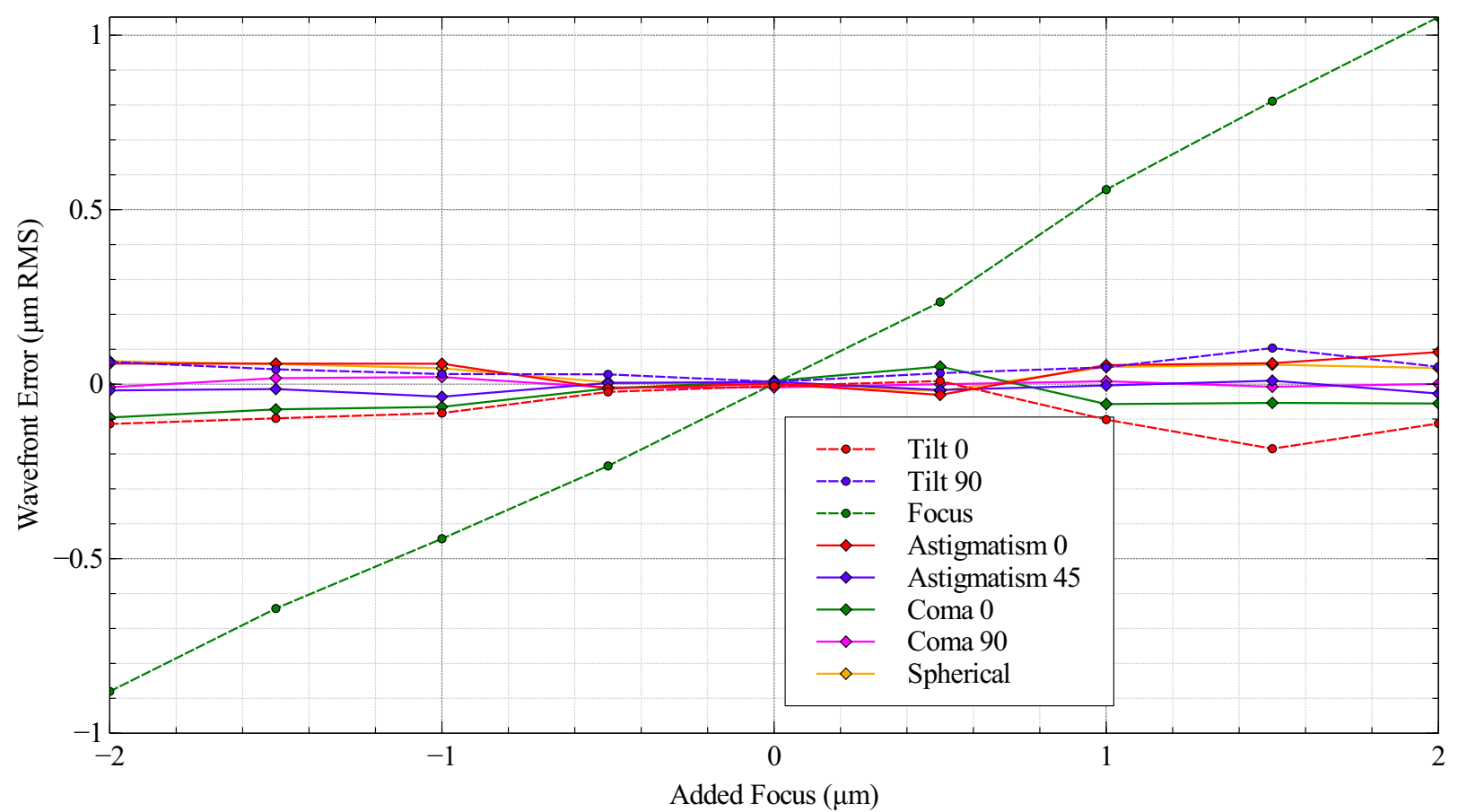

Fig 8 Effect of focus manipulation on defocus aberration (green) and on seven other high-order aberrations (according to colour in the inset).

A breakdown of the first 8 aberrations is shown in Fig. 8, where it can be seen that all these aberrations increase as defocus is applied in either direction. Defocus aberration is plotted for reference (green), and appears linear as expected. The red and blue dashed lines represent fluctuating tilt 0 and tilt 90 respectively, and seem to exhibit the highest variance.

Fig. 9 displays the RMS error for the data in Fig. 8, excluding the tilts and focus aberrations. From this graph, it is seen that the effect of focus manipulation on these aberrations is within a range between -0.1 to $0.07 \mu \mathrm{m}$ RMS. It is possible that these larger values at the extremeties of the 


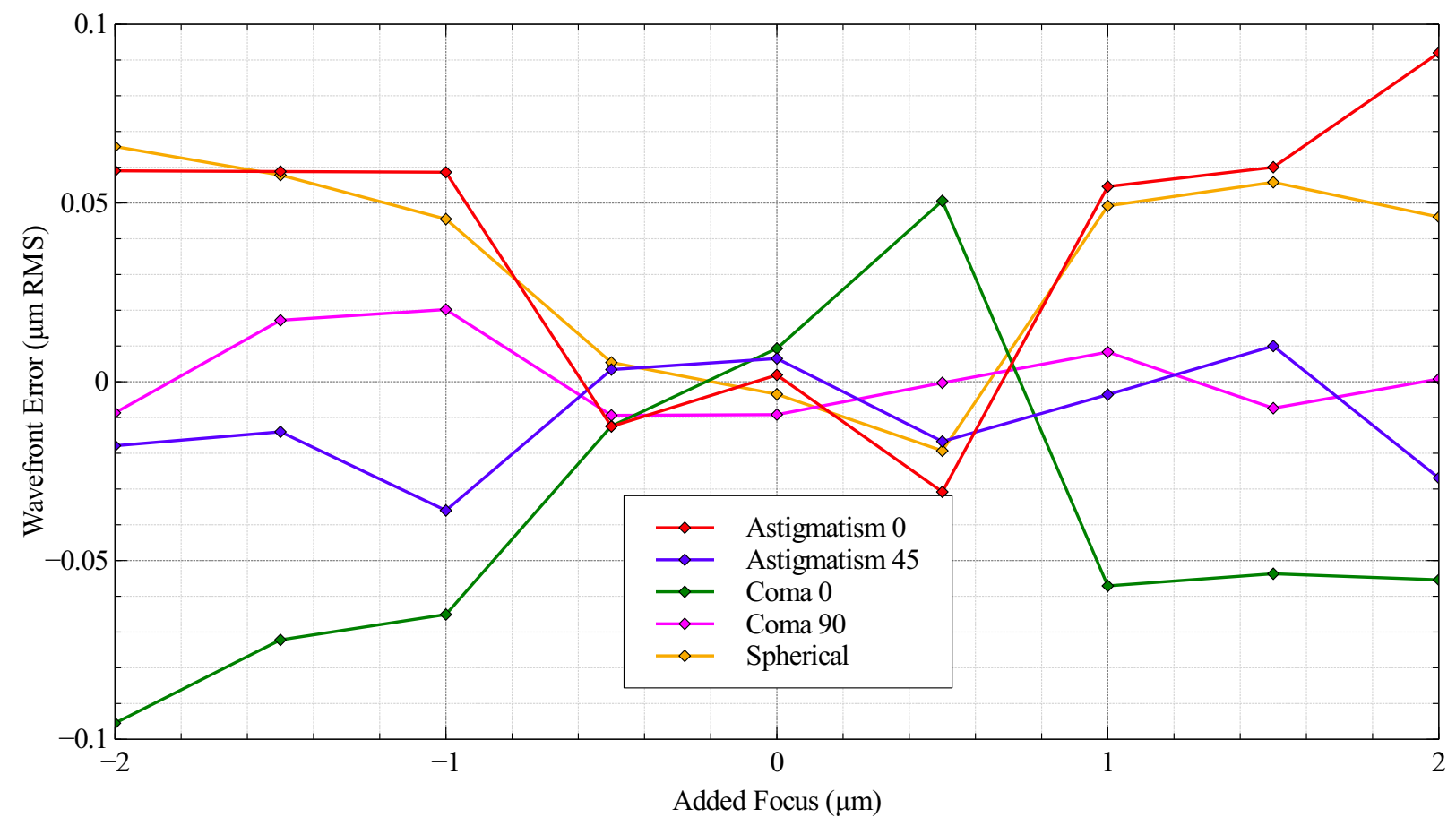

Fig 9 Magnified view of WFS RMS error showing effect of focus on wavefront excluding tilts and focus aberrations.

range are due to the limited dynamic range of the DM used. If this is the case, these can be made smaller if a second wavefront corrector is employed.

\subsection{Test procedure}

Measurements to test the procedure are conducted on a phantom target constructed using 10 layers of borosilicate glass microscope slide covers, each of thickness between 0.16 to $0.19 \mathrm{~mm}$. Each slide was bound using a single layer of thin clear tape. As the clear tape is extremely thin, distances between the slides are not resolved and so, 11 interfaces should be distinguished only. As seen in Fig. 10, even after some tilt, extra layers appear at the bottom and some layers in between the main interfaces, due to multiple reflections between the slide facets. The total thickness of the sample is approximately $2 \mathrm{~mm}$.

During live imaging, a sequence of commands is sent to the DM in rapid succession, with 
an optional time delay between each command. The magnitude of defocus aberration is swept between the limits of $\pm 4 \mu \mathrm{m}$ RMS using the DM (determining an axial range of $2 \mathrm{~mm}$ in depth). The procedure was tested with 50 steps of $40 \mu \mathrm{m}$ each at a rate of 200 commands sent to the DM per second. Oversampling by using a high number of small steps was found to produce smoother focus transition. Transversal size was maintained at $1 \mathrm{~mm}$ and the refresh of the B-scan OCT imaging was $100 \mathrm{~Hz}$.

\section{OCT imaging results}

Fig. 10A and 10C show images acquired with the focus position at respective negative $(-1 \mathrm{~mm})$ and positive $(+1 \mathrm{~mm})$ extremes, while Fig. 10B shows an intermediate unmodified state. While images are constantly being acquired we sweep the focus incrementally through 50 steps from one limit to the other, recording a video of the results. The final image in Fig. 10D is the Gabor image reconstructed using in-focus regions of all frames acquired during this sweep cycle. Fig. 10D has mostly uniform contrast throughout the range.

We can clearly see in Fig. 10A, 10B and 10C a horizontal stripe of high intensity shifting vertically in the image as the commands are applied to the DM. Fig. 10A, 10B and 10C allow an approximate visual evaluation of the $\mathrm{CG}$ profile under $\mathrm{AO}$ correction. The profile width in $10 \mathrm{~A}$ looks similar to that in 10B, where according to Fig. 2 the CG should exhibit a FWHM of $60 \mu \mathrm{m}$. The profile in 10C displays a much wider width. This suggests that other aberrations are added by the intervening layers, affecting the CG profile at $+4 \mu \mathrm{m}$ RMS more than at $-4 \mu \mathrm{m}$ RMS.

Considering the FWHM of the CG given by the minimum width of $60 \mu \mathrm{m}$ and given the axial range of $2 \mathrm{~mm}$, a minimum value of 33 is obtained for the ratio $\mathrm{R}$. We used an experimental value of $\mathrm{R}_{\max }=50$ for oversampling. 


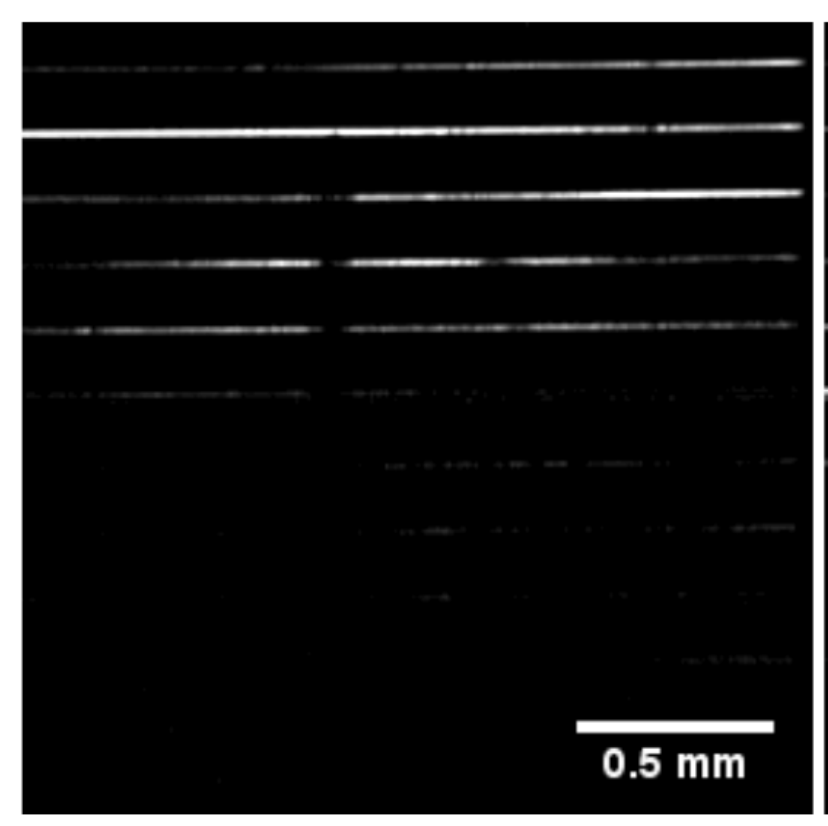

(A)

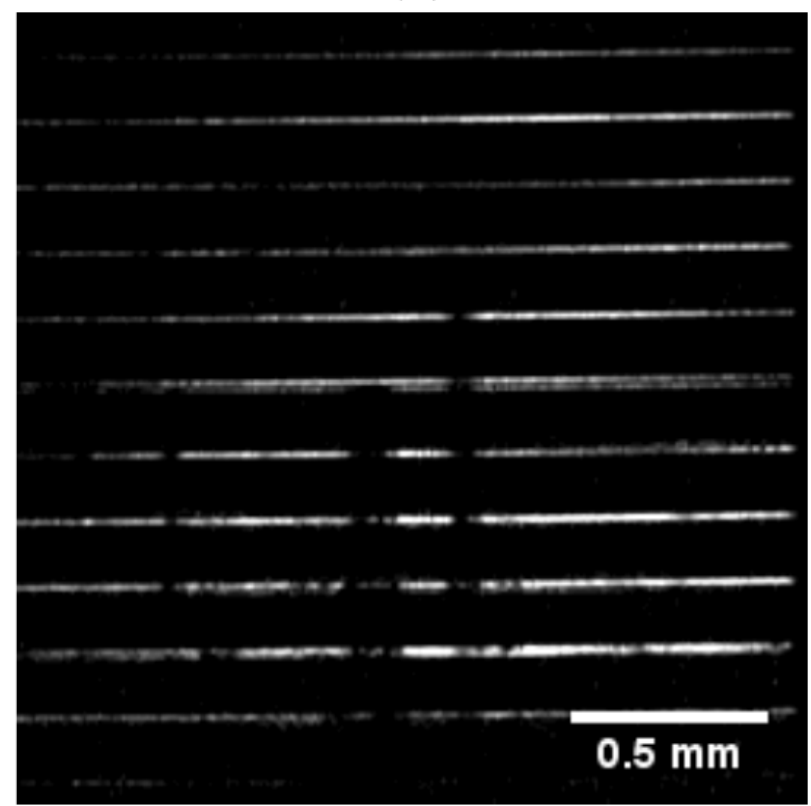

(C)

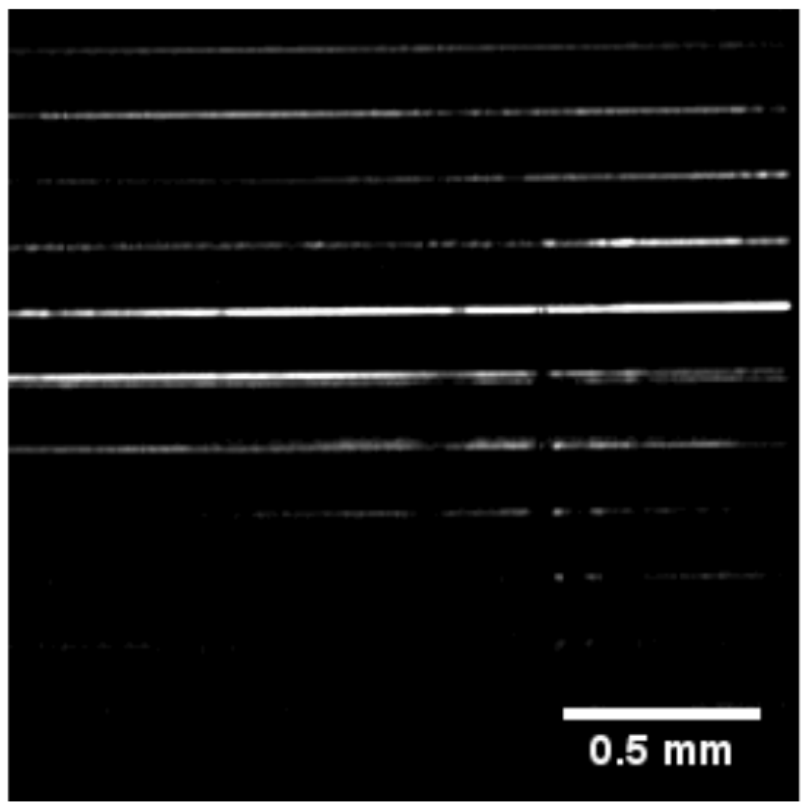

(B)

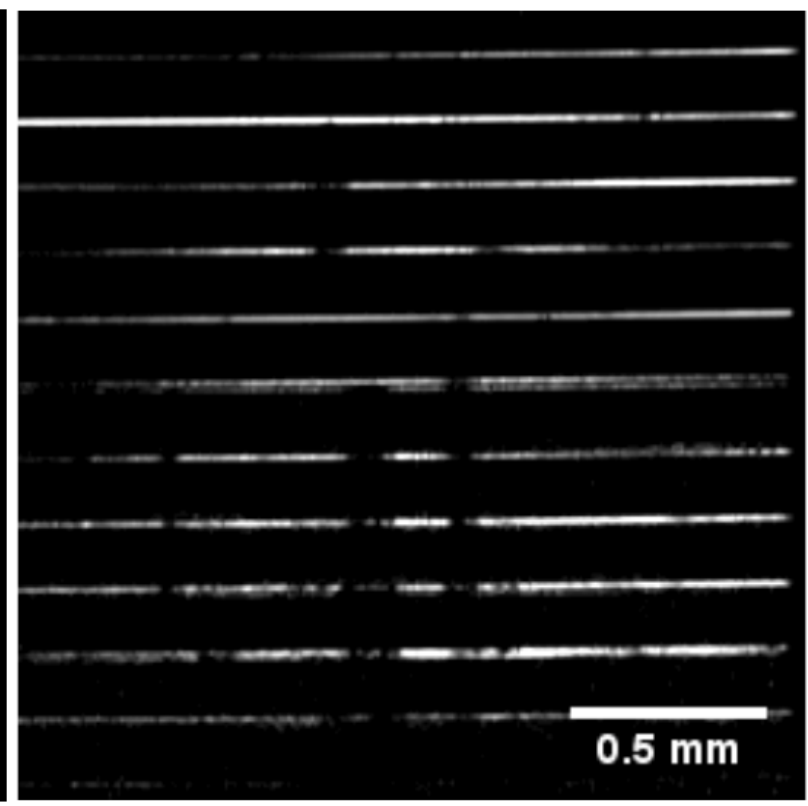

(D)

Fig 10 Images acquired of a phantom constructed from ten $0.17 \mathrm{~mm}$ thick glass slides (bound top and bottom with sellotape of similar thickness) with focus set to -4 (A), 0 (B) and 4 (C) covering a total range of 2 mm. Final image (D) is synthesized through extraction of in-focus regions from a stack of images acquired with focus shifted incrementally. 


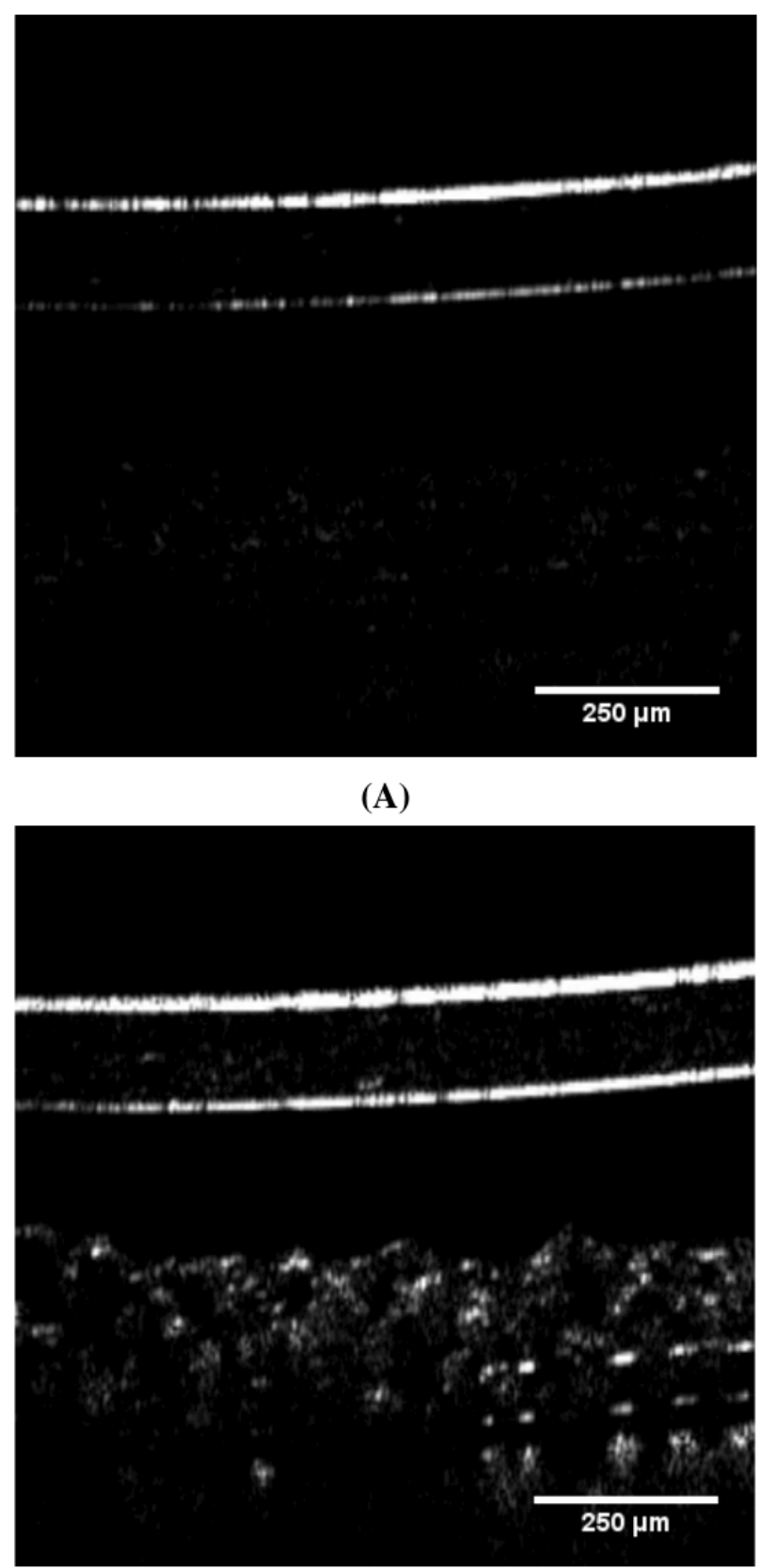

(C)

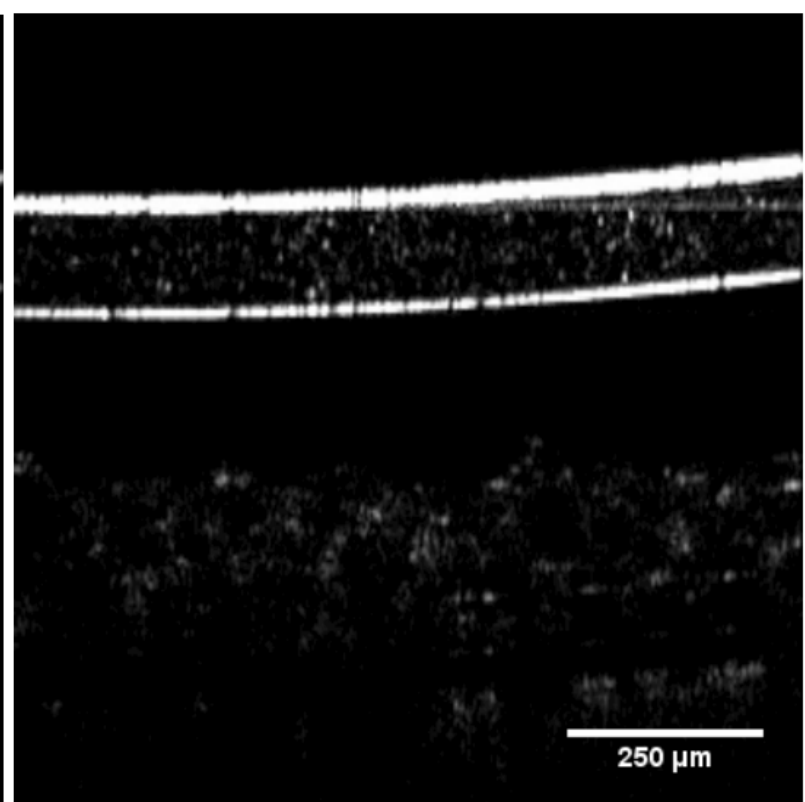

(B)

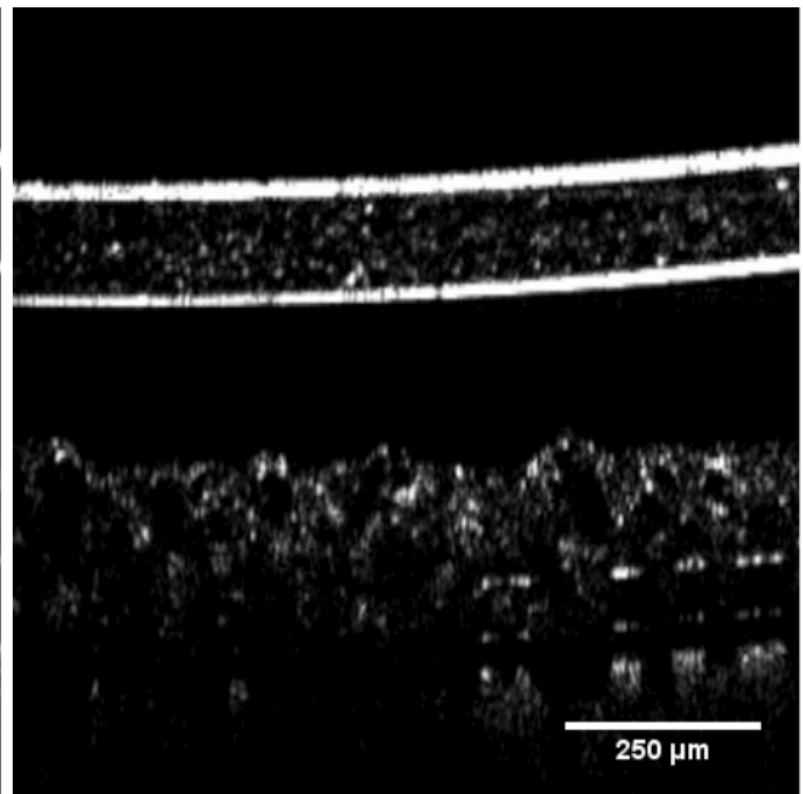

(D)

Fig 11 OCT B-scan images of an infra-red card, with focus set to -1.5 (A), 0 (B), and 1.5 (C) covering a range of 0.75 $\mathrm{mm}$. The final image (D) is the composite Gabor image. 
The procedure was repeated using an infra-red card as the object, with the DM configured to sweep $\pm 375 \mu \mathrm{m}$ (total axial range of $0.75 \mathrm{~mm}$ ). Fig. 11D is assembled by stitching the in-focus regions of images acquired during the focus sweep (Fig. 11A through 11C). It can be observed that the Gabor image in 11D exhibits the details in both the superficial layers (shown in Fig. 11A), intermediate layers (Fig. 11B) and deeper layers (Fig. 11C).

To verify that this procedure is suitable for in-vivo imaging of live samples we performed the same experiment on a wild-type Drosophila larvae. Several larvae were chosen at varying stages of development and secured using strong double-sided tape. All larvae are highly active and move constantly, potentially compromising the integrity of acquired images. This time we reduce the focus sweep to $\pm 500 \mu \mathrm{m}$, covering a total axial range of $1 \mathrm{~mm}$. Total power to the sample was measured to be $2.3 \mathrm{~mW}$, which is well within safety range for a scanning beam at $1060 \mathrm{~nm}$.

Raw images from a Drosophila larvae taken with the focus shifted at $-0.5 \mathrm{~mm}, 0$ and $0.5 \mathrm{~mm}$ in air are presented in Fig. 12A, 12B and 12C respectively. Fig. 12D shows the final Gabor filtered synthesized image, produced by compounding the regions of maximum intensity in each image from a stack of images recorded during a full sweep cycle.

Finally, the procedure was tested upon the thumb of a volunteer using an axial sweep range of $1 \mathrm{~mm}$. Fig. 13A shows an image taken with static focus in the base state. The surface of the thumb is highly visible, as is the general position and structure of two sweat ducts. The distinctive spiral structure of the sweat duct is well documented in literature. ${ }^{21}$ Fig. 13B shows the thumb with focus swept deeper into tissue; not only have the sweat ducts become more clearly defined, but also the deeper structures of the epidermis.

To produce the synthesized images in Fig. 10D, 11D, 12D and in Fig. 13B, ImageJ was used post-acquisition. Given the speed of the image acquisition and DM command rate it would be a 


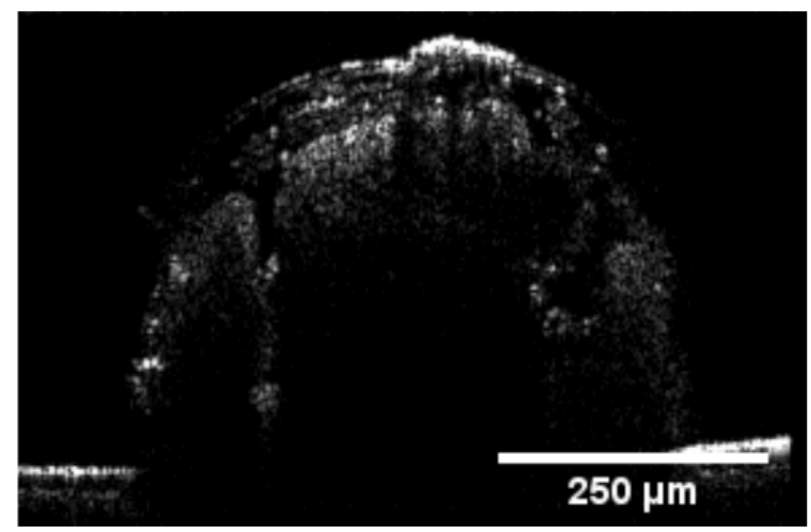

(A)

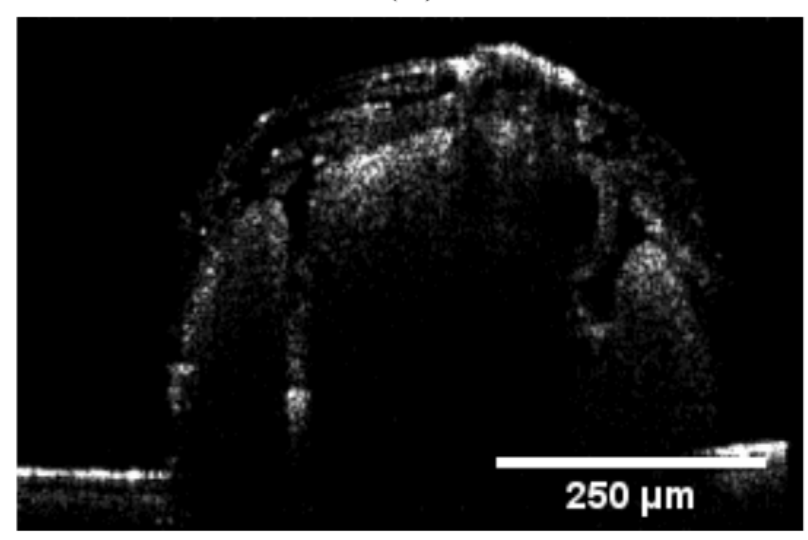

(C)

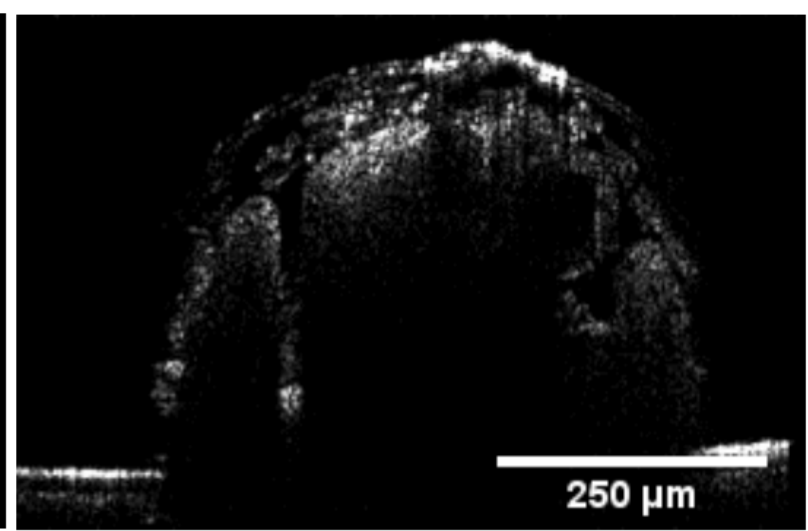

(B)

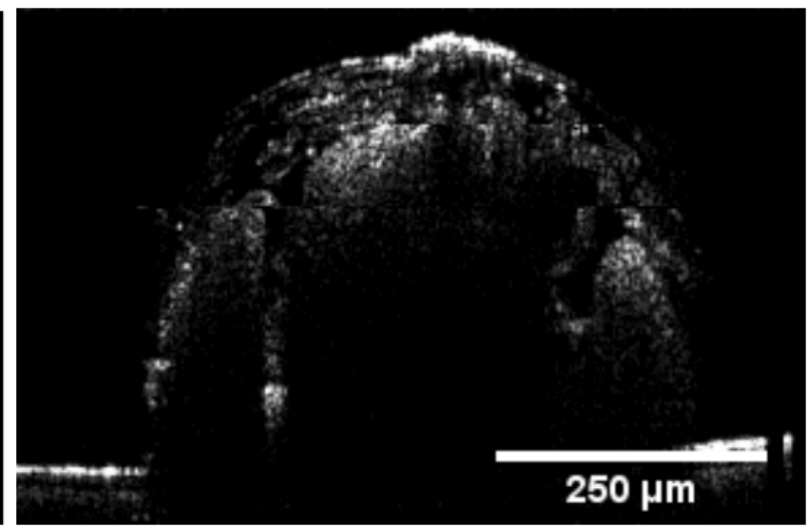

(D)

Fig 12 B-scan-OCT images of in-vivo Drosophila with focus at -1 (A), 0 (B), and 1 (C) covering a total range of 1 $\mathrm{mm}$ axial range measured in air. Final image (D) is synthesized through extraction of in-focus regions from a stack of images acquired with focus shifted incrementally.

simple evolutionary step to perform the splicing of cropped images live.

Artifacts may form in the synthesized image should the sample move during acquisition, which is a common problem in live imaging. One avenue of compensation is to increase the rate at which commands are sent to the DM, either by adjusting the delay between each command or simply reducing the number of incremental focus shifts to the minimum required for sufficient sampling as determined by $\mathrm{R}$. 


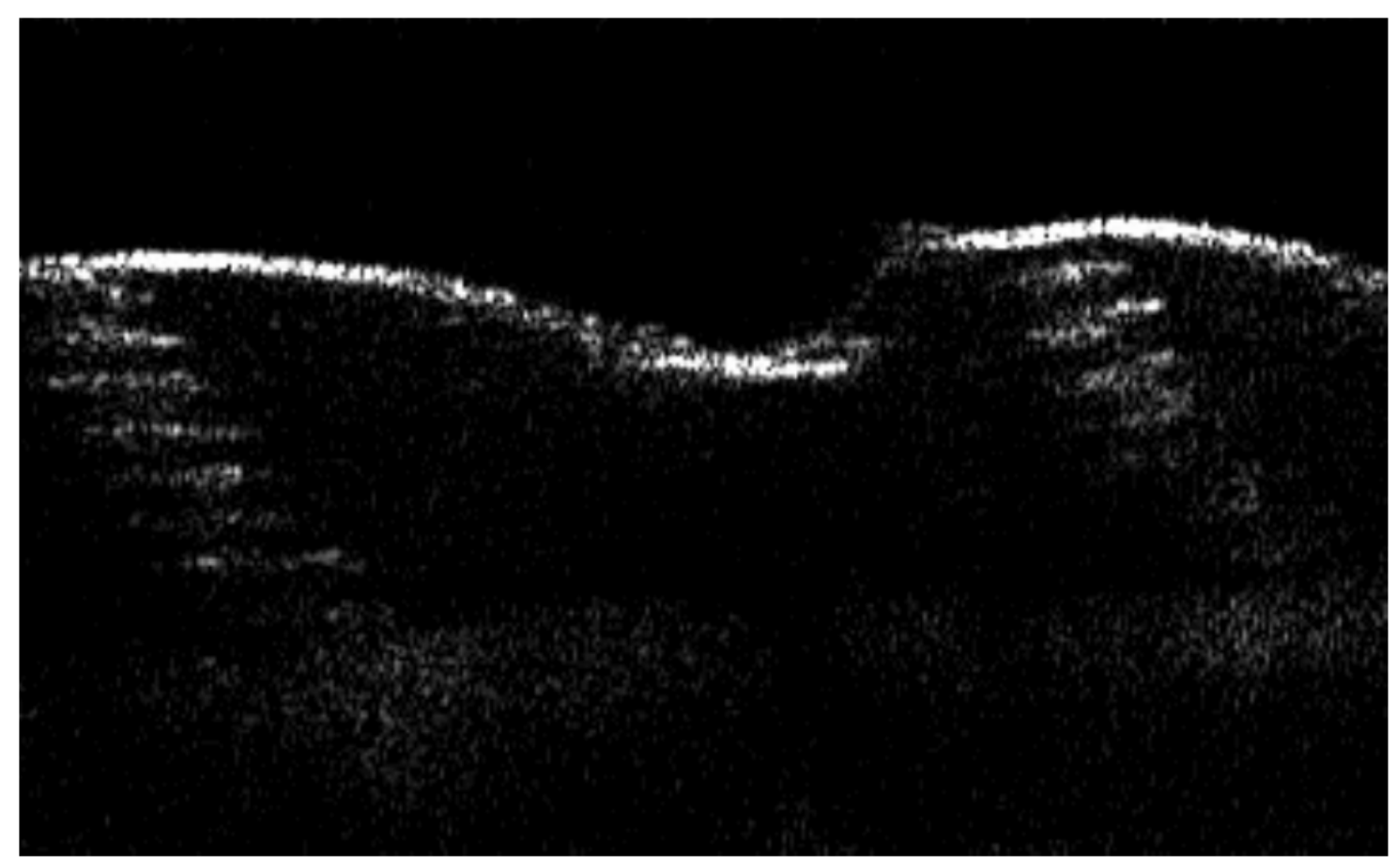

(A)

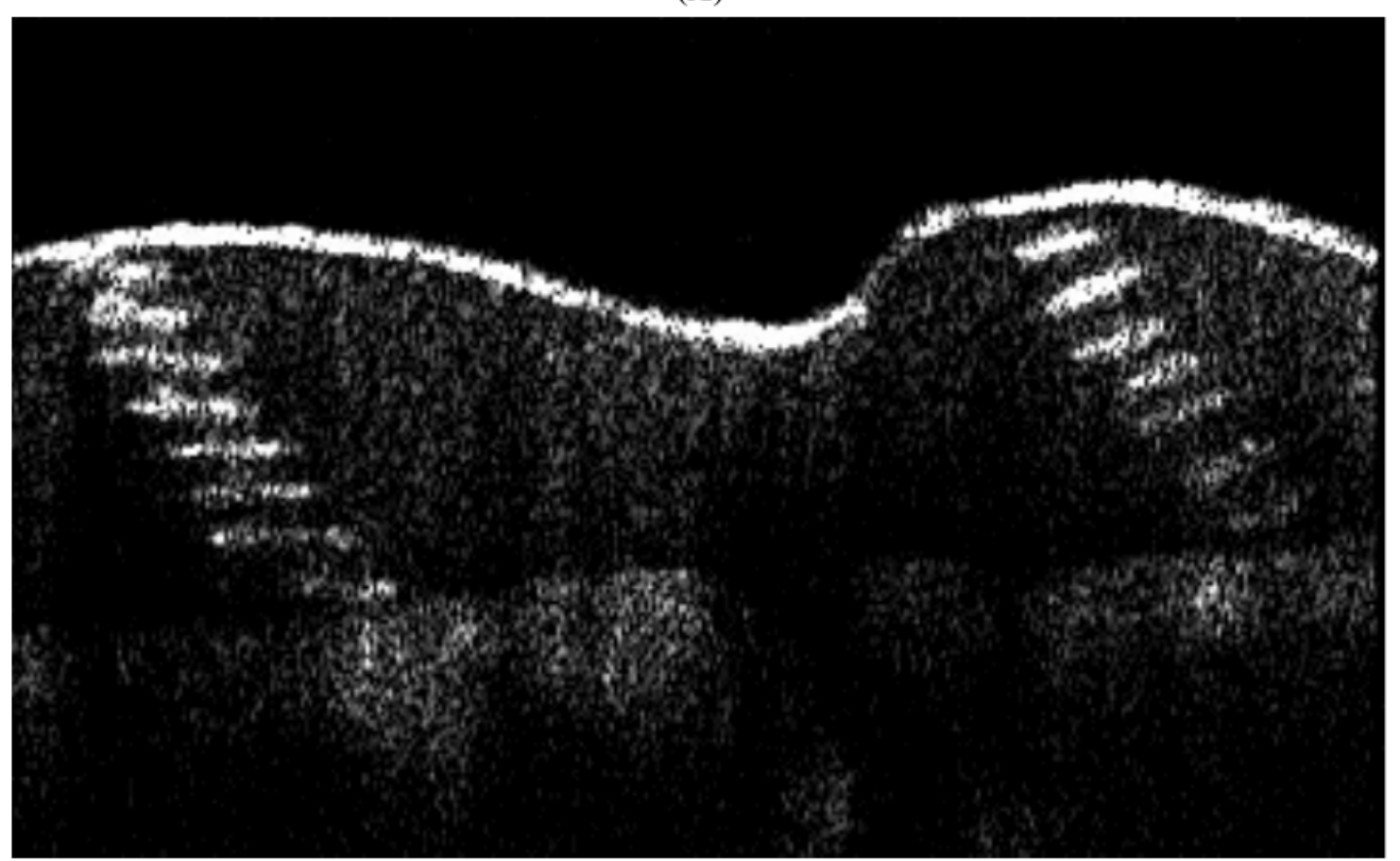

(B)

Fig 13 In-vivo OCT B-scan images acquired of thumb with fixed focus (A) at the surface and after Gabor splicing (B). Visible are the distinctive spiral structures of the sweat ducts, and the deeper tissues are enhanced in (B) due to focus sweep. Vertical scale: $2 \mathrm{~mm}$ measured in air 


\section{Conclusions}

The paper demonstrates that fine control over the curvature of a DM can be employed to perform Gabor splicing and yield better uniformity in terms of signal intensity throughout depth in a SSOCT configuration.

This technique presents a clear advantage over possible implementations of focus control using a liquid crystal lens added to an AO configuration, as there exists potential to perform the focus shift whilst maintaining closed loop corrections using the DM. Even without updating the correction, the loss of signal up to half of its maximum can be tolerated within a significant axial scanning range of more than $2 \mathrm{~mm}$. In addition, we would emphasize the benefit and sophistication of the $\mathrm{DM}$ as we are able to make several hundreds of incremental high-precision focus adjustments every second.

However, a number of shortcomings should be considered in practice. In addition to variation in sensitivity with defocus as shown in Fig. 3, there is a marked lateral shift of the image, in both vertical and horizontal direction. This was quantified here using confocal images of a USAF target, observing a lateral shift of approximately $2 \mu \mathrm{m}$ per each $1 \mu \mathrm{m}$ defocus applied to the DM. Large images were used here, where such lateral shifts could be ignored. Small size images however would require correction for the lateral shifts.

The lateral shifts induced by deforming the DM were stable in time, however the dependence of such shift to the object tilt prevents a universal compensation algorithm. An individual case solution can be implemented by first tracking the lateral shift for a given orientation and then correcting by applying corresponding bias values to the two transversal scanners. Knowing that $100 \mathrm{mV}$ determines an image size of $70 \mu \mathrm{m}$, a $2 \mu \mathrm{m}$ correction would require approximately $3 \mathrm{mV}$ 
bias to each driver signal applied to the two transversal scanners. This would have some limited applicability, as individual layers with different inclinations inside the tissue will register different lateral shifts.

In the case presented here, a single DM is used to compensate for all aberrations and then used to manipulate defocus. Advanced designs may use multiple DMs to great effect, for example one DM may exclusively control tilts to compensate lateral shift. Alternatively, one DM could be set to continuously run closed loop correction (excluding focus control) whilst the second DM shifts the focus. This would eliminate the procedure of pre-processing the files for focus manipulation. Utilization of two deformable mirrors may improve the correction of the wavefront when limited by the dynamic range of a single corrector.

It can also be noticed by inspecting Fig. 10D, 11D, 12D and in Fig. 13B that the CG stripe can be seen moving axially with defocus aberration in Fig. 10D, but less in Fig. 11D and much less in Fig. 12D and Fig. 13B . This is a clear indication of enlargement of the CG profile due to aberrations created by the intermediate interfaces up to the depth of interest. Also, the variation of brightness amongst the sequence of images versus focus is not as sharp as illustrated by the profile in Fig. 3. This is another consequence of extra aberrations than those measured in Fig. 8 and 9; scattering caused by intermediate micro interfaces and scattering centres in the Drosophila and the thumb alter the shape and peak of the CG profile. This also shows that the correction determined by the WFS based on the dominant signal collected from the surface of a scattering sample is not so useful for imaging inside the volume of the sample. 


\section{Acknowledgments}

C. Costa, A. Bradu and A. Podoleanu acknowledge the support of the European Research Council (http://erc.europa.eu), grant 249889. A. Podoleanu also acknowledges support from the Biomedical Research Centre at Moorfields Eye Hospital NHS Foundation Trust and UCL Institute of Ophthalmology.

\section{References}

1 M. Booth, "Adaptive Optics for High-resolution Microscopy," in Imaging and Applied Optics, J. Christou and D. Miller, Eds., OM1A.2, OSA, (Washington, D.C.) (2013).

2 A. Dubra, Y. Sulai, J. L. Norris, R. F. Cooper, A. M. Dubis, D. R. Williams, and J. Carroll, "Noninvasive imaging of the human rod photoreceptor mosaic using a confocal adaptive optics scanning ophthalmoscope," Biomedical optics express 2(7), 1864-1876 (2011).

3 E. J. Fernandez, L. Vabre, B. Hermann, A. Unterhuber, B. Povazay, and W. Drexler, “Adaptive optics with a magnetic deformable mirror: applications in the human eye," Optics Express 14, 8900 (2006).

4 R. J. Zawadzki, S. M. Jones, S. S. Olivier, M. Zhao, B. A. Bower, J. A. Izatt, S. Choi, S. Laut, and J. S. Werner, "Adaptive-optics optical coherence tomography for high-resolution and high-speed 3D retinal in vivo imaging," Optics Express 13, 8532 (2005).

5 B. Cense, E. Koperda, J. M. Brown, O. P. Kocaoglu, W. Gao, R. S. Jonnal, and D. T. Miller, "Volumetric retinal imaging with ultrahigh-resolution spectral-domain optical coherence tomography and adaptive optics using two broadband light sources," Optics Express 17, 4095 (2009). 
6 W. Wieser, B. R. Biedermann, T. Klein, C. M. Eigenwillig, and R. Huber, "Multi-megahertz OCT: High quality 3D imaging at 20 million A-scans and 4.5 GVoxels per second.," Optics express 18, 14685-704 (2010).

7 W. Choi, B. Potsaid, V. Jayaraman, B. Baumann, I. Grulkowski, J. J. Liu, C. D. Lu, A. E. Cable, D. Huang, J. S. Duker, and J. G. Fujimoto, "Phase-sensitive swept-source optical coherence tomography imaging of the human retina with a vertical cavity surface-emitting laser light source.," Optics letters 38, 338-40 (2013).

8 M. Mujat, R. D. Ferguson, A. H. Patel, N. Iftimia, N. Lue, and D. X. Hammer, "High resolution multimodal clinical ophthalmic imaging system.," Optics express 18, 11607-21 (2010).

9 B. Potsaid, B. Baumann, D. Huang, S. Barry, A. E. Cable, J. S. Schuman, J. S. Duker, and J. G. Fujimoto, "Ultrahigh speed 1050nm swept source/Fourier domain OCT retinal and anterior segment imaging at 100,000 to 400,000 axial scans per second.," Optics express 18, 20029-48 (2010).

10 Z. Ding, H. Ren, Y. Zhao, J. S. Nelson, and Z. Chen, "High-resolution optical coherence tomography over a large depth range with an axicon lens," Optics Letters 27, 243 (2002).

11 R. A. Leitgeb, M. Villiger, A. H. Bachmann, L. Steinmann, and T. Lasser, "Extended focus depth for Fourier domain optical coherence microscopy," Optics Letters 31(16), 2450 (2006).

12 D. Lorenser, C. Christian Singe, A. Curatolo, and D. D. Sampson, "Energy-efficient lowFresnel-number Bessel beams and their application in optical coherence tomography.," Optics letters 39, 548-51 (2014).

13 J. P. Rolland, P. Meemon, S. Murali, K. P. Thompson, and K.-s. Lee, “Gabor-based fusion technique for Optical Coherence Microscopy.," Optics Express 18(4), 3632-3642 (2010). 
14 B. Qi, A. Phillip Himmer, L. Maggie Gordon, X. Victor Yang, L. David Dickensheets, and I. Alex Vitkin, "Dynamic focus control in high-speed optical coherence tomography based on a microelectromechanical mirror," Optics Communications 232, 123-128 (2004).

15 M. Hughes and A. G. Podoleanu, "A simplified dynamic focus method for time domain OCT," 46(1), 1-6 (1991).

16 K. Sasaki, K. Kurokawa, S. Makita, and Y. Yasuno, "Extended depth of focus adaptive optics spectral domain optical coherence tomography.," Biomedical optics express 3, 2353-70 (2012).

17 C. Torti, B. Povazay, B. Hofer, A. Unterhuber, J. Carroll, P. K. Ahnelt, and W. Drexler, "Adaptive optics optical coherence tomography at 120,000 depth scans/s for non-invasive cellular phenotyping of the living human retina.," Optics express 17, 19382-400 (2009).

18 A. Dubra and Y. Sulai, "Reflective afocal broadband adaptive optics scanning ophthalmoscope," Biomedical optics express 2(6), 1757-1768 (2011).

19 A. G. Podoleanu and A. Bradu, "Master-slave interferometry for parallel spectral domain interferometry sensing and versatile 3D optical coherence tomography.," Optics express 21, 19324-38 (2013).

20 E. Ödlund, H.-F. Raynaud, C. Kulcsár, F. Harms, X. Levecq, F. Martins, N. Chateau, and A. G. Podoleanu, "Control of an electromagnetic deformable mirror using high speed dynamics characterization and identification," Applied Optics 49, G120 (2010).

21 M. Ohmi, M. Tanigawa, Y. Wada, and M. Haruna, "Dynamic analysis for mental sweating of a group of eccrine sweat glands on a human fingertip by optical coherence tomography.," Skin research and technology : official journal of International Society for Bioengineering 
and the Skin (ISBS) [and] International Society for Digital Imaging of Skin (ISDIS) [and]

International Society for Skin Imaging (ISSI) 18, 378-83 (2012).

\section{List of Figures}

1 Schematic of the AO/SS-OCT system with imaging at $1060 \mathrm{~nm}$ and wavefront sensing plus confocal microscopy at $830 \mathrm{~nm}$.

2 Chart showing how the confocal profile shifts in depth and changes shape as we add 1 and $2 \mu \mathrm{m}$ RMS focus.

3 Chart showing decay in signal intensity with added focus

4 High contrast confocal image of the USAF target showing the smallest $2 \mu \mathrm{m}$ bars at the bottom. Two sets of three bars are shown oriented horizontally and vertically. Image size is $250 \times 250$ pixels covering an actual area of $70 \times 70 \mu \mathrm{m}^{2}$ on the target.

5 Effect of defocus on the image acquired from the USAF target, using the same area as in Fig. 4. The original image (C) is repeated in (D) and placed alongside images taken with -2 (A), -1 (B), 1 (E) and 2 (F) $\mu \mathrm{m}$ RMS focus added to the DM.

6 Images of the USAF target obtained using the DM deformed to create $3 \mu \mathrm{m}$ (B) and $4 \mu \mathrm{m}$ (C) RMS defocus, with the original $0 \mu \mathrm{m}$ focus image (A) for comparison.

7 RMS wavefront error as focus is manipulated. Total error: green region. Error excluding focus: blue region. Error excluding tilts and focus: red region.

8 Effect of focus manipulation on defocus aberration (green) and on seven other high-order aberrations (according to colour in the inset). 
9 Magnified view of WFS RMS error showing effect of focus on wavefront excluding tilts and focus aberrations.

10 Images acquired of a phantom constructed from ten $0.17 \mathrm{~mm}$ thick glass slides (bound top and bottom with sellotape of similar thickness) with focus set to -4 (A), 0 (B) and 4 (C) covering a total range of $2 \mathrm{~mm}$. Final image (D) is synthesized through extraction of in-focus regions from a stack of images acquired with focus shifted incrementally.

11 OCT B-scan images of an infra-red card, with focus set to -1.5 (A), 0 (B), and 1.5 (C) covering a range of $0.75 \mathrm{~mm}$. The final image (D) is the composite Gabor image.

12 B-scan-OCT images of in-vivo Drosophila with focus at -1 (A), 0 (B), and 1 (C) covering a total range of $1 \mathrm{~mm}$ axial range measured in air. Final image (D) is synthesized through extraction of in-focus regions from a stack of images acquired with focus shifted incrementally.

13 In-vivo OCT B-scan images acquired of thumb with fixed focus (A) at the surface and after Gabor splicing (B). Visible are the distinctive spiral structures of the sweat ducts, and the deeper tissues are enhanced in (B) due to focus sweep. Vertical scale: $2 \mathrm{~mm}$ measured in air 